\title{
THE
}

\section{Adolescent and caregiver perception of family functioning: Relation to suicide ideation and attempts}

Jessica M. Lipschitz

University of Rhode Island

Shirley Yen

Lauren M. Weinstock

Anthony Spirito

Follow this and additional works at: https://digitalcommons.uri.edu/psy_facpubs

This is a pre-publication author manuscript of the final, published article.

Creative Commons License

(c) (1)(9)

This work is licensed under a Creative Commons Attribution-Noncommercial-No Derivative Works 4.0 License.

\section{Citation/Publisher Attribution}

Lipschitz, J. M., Yen, S., Weinstock, L. M., \& Spirito, A. (2012). Adolescent and caregiver perception of family functioning: Relation to suicide ideation and attempts. Psychiatry Research, 200(2-3), 400-403. doi: 10.1016/j.psychres.2012.07.051

Available at: https://doi.org/10.1016/j.psychres.2012.07.051

This Article is brought to you for free and open access by the Psychology at DigitalCommons@URI. It has been accepted for inclusion in Psychology Faculty Publications by an authorized administrator of DigitalCommons@URI. For more information, please contact digitalcommons-group@uri.edu. 


\title{
Adolescent and caregiver perception of family functioning: relation to suicide ideation and attempts
}

\author{
Jessica M. Lipschitz, \\ Department of Psychology, University of Rhode Island, Kingston, Rhode Island \\ Shirley Yen, \\ Department of Psychiatry and Human Behavior, Alpert Medical School of Brown University, \\ Providence, RI
}

Lauren M. Weinstock, and Department of Psychiatry and Human Behavior, Alpert Medical School of Brown University, Providence, RI

\begin{abstract}
Anthony Spirito
Department of Psychiatry and Human Behavior, Alpert Medical School of Brown University, Providence, RI
\end{abstract}

\section{Abstract}

This study assessed the relationship between perceived family functioning and two indicators of suicidality in an adolescent sample. A total of 103 adolescents psychiatrically hospitalized for suicidal ideation and/or behavior completed a battery of self-report questionnaires assessing family functioning, negative affectivity, suicidal ideation in the past week assessed by Beck's Scale for Suicide Ideation (BSS; Beck et al., 1979), and past suicide attempts. Participants' primary caregivers also completed a questionnaire on family functioning. A paired samples t-test evaluated overall discrepancy between adolescent- and caregiver-reported family functioning. Linear and logistic regression models were used to evaluate the simple associations between perceived family functioning and suicidal indicators (BSS and previous attempt status), as well as the associations between perceived family functioning and suicidal indicators after controlling for negative affect. Results indicated that adolescents' ratings of family functioning were significantly worse than caregivers' ratings, and positively associated with BSS scores and a history of suicide attempt. When negative affect was controlled for, adolescent-reported family functioning was significantly associated with BSS, but only trended toward significance with attempt status. Findings suggest that adolescents' perceptions of family functioning may be a key contributing factor to suicidal ideation in adolescents with psychiatric disorders.

\section{Keywords}

suicide; family functioning; negative affect; adolescence

\footnotetext{
(C) 2012 Elsevier Ireland Ltd. All rights reserved.

Correspondence concerning this article should be addressed to: Jessica M. Lipschitz, Department of Psychology, University of Rhode Island, 2 Chafee Road, Kingston, RI, USA 02881.jmlipschitz@gmail.com.

Publisher's Disclaimer: This is a PDF file of an unedited manuscript that has been accepted for publication. As a service to our customers we are providing this early version of the manuscript. The manuscript will undergo copyediting, typesetting, and review of the resulting proof before it is published in its final citable form. Please note that during the production process errors may be discovered which could affect the content, and all legal disclaimers that apply to the journal pertain.
} 


\section{Introduction}

In the United States, suicide is the third leading cause of death in the 15-24 age range. Of even greater concern is that the rate of suicide deaths and suicidal behaviors in adolescents has risen approximately $11 \%$ since 1980 (CDC, 2002). In 2009, a CDC survey of high school students nationwide found that $13.8 \%$ had thought about attempting suicide in the past 12 months and 6.3\% indicated that they made a suicide attempt (CDC, 2010).

Anxiety and mood disorders are leading risk factors for suicidal behavior. A substantial body of research indicates that presence and severity of depressive disorders are significantly associated with suicidal acts in adolescents (Shaffer et al., 1996; Muehlenkamp \& Gutierrez, 2007; Nrugham et al., 2008; Goldston et al., 2009). This is also the case with anxiety disorders. For example, Liu et al. (2006) found significantly more anxiety symptoms in suicidal adolescents than in nonsuicidal adolescent psychiatric patients. In addition, repeat attempts are positively associated with incidence of anxiety disorders in suicidal adolescents (Miranda et al., 2008; Goldston et al., 2009).

However, it remains unclear why some individuals with mood and anxiety disorders attempt suicide whereas others do not. The intra-familial environment has been proposed as a factor that may be responsible for different suicide outcomes among depressed patients. This variable is of particular interest because, unlike many other predictors, it is modifiable through treatment. Studies on adult populations find that significant discrepancies between patient and caregiver perceptions of family functioning exist in suicide attempters but not ideators or non-suicidal patients (Keitner et al., 1987; McDermut et al., 2001).

Whereas studies consistently find that suicidal behavior in adolescents is associated with family dysfunction (e.g., King et al., 1993; Martin et al., 1995; Brinkman-Sull et al., 2000; Bridge et al., 2006), there is limited research on caregiver-child discrepancies and how they relate to suicidal outcomes. Moreover, research evaluating the association between family functioning and suicidal behavior, while controlling for the effects of depression and anxiety or negative affect, is scant. The limited research that has been published finds that including factors such as depression severity in the predictive model as a covariate eliminates the significance of variables representing family functioning in a predictive model of suicide (King, et al., 1993; Brinkman-Sull, et al., 2000).

This study has several aims. First, we evaluated the discrepancy between caregiver and adolescent perceptions of family functioning in a sample of suicidal adolescents. Second, we evaluated adolescent-reported, caregiver-reported, and the discrepancy between adolescent and caregiver reports of family functioning as predictors of suicidal ideation and behavior. Finally, we examined whether perceptions of family functioning were significantly associated with severity of suicidal ideation or history of suicide attempts when controlling for negative affect. It was hypothesized that child and caretaker family functioning ratings would be significantly different and that perceptions of family functioning would be associated with suicidal ideation and attempt status even when controlling for negative affect.

\section{Methods}

\subsection{Participants}

Participants were recruited for a naturalistic, follow-up study on the course of emotional functioning in suicidal adolescents over the 6-month period after a psychiatric hospitalization. Only participants with complete case data on the relevant self-report 
measures at baseline are included in this report. Procedures were approved by hospital and university Institutional Review Boards.

Participants in the parent study consisted of 119 adolescents and their legal guardians/ primary caregivers recruited from a psychiatric hospital in the Northeastern United States. Of these, 103 adolescents and 88 caregivers provided complete family assessment data and are, therefore, included in the present study. Adolescents were recruited on the basis of being hospitalized for some suicidal risk (i.e., attempts, self-injury with suicidal intent, and/ or ideation), as identified through chart review of consecutive admissions. Potential participants were approached by a research assistant during visiting hours. Parental consent and adolescent assent were required to proceed with the intake assessment.

Of the 103 suicidal adolescent patients enrolled in this study who provided family assessment data, $30.1 \%$ were male and $69.9 \%$ were female. The sample was $79.4 \%$ Caucasian, 9.8\% African American, 2.0\% were American Indian/Alaskan Native, and 8.8\% fell outside of these categories. Participants' ages ranged from 13 to 18 years old with a mean age of $15.36(S D=1.36)$. The majority of the adolescent participants lived with one or more biological parent (85.4\%). Nearly two thirds of the sample (60.3\%) reported having made a suicide attempt. Diagnosis and other clinical characteristics of the full sample are described in Yen et al. (under review).

\subsection{Assessment Instruments}

2.21 Family Assessment Device General Functioning Subscale (FAD; Epstein et al., 1983)—The 12-item general functioning subscale of the Family Assessment Device (FAD; Epstein et al., 1983) was used as a self-report measure of family functioning. Subscale scores on the FAD range from 1 to 4 , with higher scores representing poorer family functioning. A clinical cutoff score of 2.0 has been specified to identify families that fall within the range of unhealthy functioning on the FAD general functioning subscale (Miller et al., 1985). The FAD has been used widely, has established construct validity (Miller et al., 1985), and previous research supports the factorial validity of its subscales (Kabacoff et al., 1990). Additionally Epstein et al. (1983) found support of concurrent validity with other scales thought to measure related constructs as well as discriminant validity of the FAD's ability to distinguish individuals from families presenting clinically and those from families not presenting clinically. The general functioning subscale of the FAD has been used in prior research as a brief measure of overall family functioning (e.g., Byles et al., 1988; Uebelacker et al., 2006; Weinstock and Miller, 2010). Internal consistency of the FAD general functioning subscale in the present study was strong ( $a=$ 0.91 for adolescents; $\alpha=0.88$ for caregivers).

2.22 Beck Scale for Suicide Ideation (BSS; Beck et al., 1979)—The BSS is a 21item self-report questionnaire intended to measure suicidal ideation. Principal component analysis of the clinician-rated version of the BSS, the Scale for Suicide Ideation (Holi et al., 2005), suggests that it evaluates three types of suicidal ideation: active suicidal desire, passive suicidal desire, and specific plans for suicide. Participants respond to items on a 3point scale that ranges from 0 (not present) to 2 (highest severity of suicidal ideation). Thus higher scores suggest greater suicidal intent. Excellent internal $(a=0.89-0.96)$ and interrater $(r=0.83)$ reliability as well as sound construct validity have been reported (Beck et al., 1979). Internal consistency in the current study was excellent $(a=0.92)$.

\subsection{Schedule for Affective Disorders and Schizophrenia for School Aged Children - Present and Lifetime Versions (K-SADS-PL; Kaufman et al., 1997)—} The K-SADS-PL is a semi-structured diagnostic interview, which provides a reliable and 
valid assessment of DSM-IV psychopathology in children and adolescents. This interview also contains questions to assess past history of suicide attempts, including details regarding the attempt, ratings of intent to die, and medical threat of the suicide attempt. The K-SADSPL was administered with adolescent and caregiver participants individually. In the present set of analyses, we use only data pertaining to past history of suicidal behavior as reported by the adolescent, as parents were not always aware of past attempts. Suicide attempt status was operationalized dichotomously.

2.24 Negative Affectivity Self Statement Questionnaire (NASSQ; Ronan et al., 1994)-The NASSQ is a 39-item self-report questionnaire used to evaluate self-statements that correspond with negative affect in children and young adults over the previous week. Participants responded to negative self-statement items using a 5-point Likert-type scale ranging from 1 (not at all) to 5 (all the time). Higher scores indicate greater self-referent negative cognitive experiences. Ronan et al. (1994) found support for the concurrent and construct validity of this measure. For example, Pearson correlations between the anxious and depressive self-statements and well-accepted measures of anxiety and depression, such as the State Trait Anxiety Inventory for Children and the Children's Depression Inventory, were significant. Ronan et al. (1994) also found appropriate Spearman-Brown $(r=0.94)$, alpha $(a=0.96)$ and test-retest $(r=.78)$ reliability estimates. Internal consistency in this data was excellent $(a=.96)$.

\subsection{Data Analysis}

Analysis involved three steps. The first step examined the nature of the differences between adolescent and caregiver ratings of family functioning. A paired samples t-test was conducted to compare adolescent- and caregiver-reported FAD. The second step involved evaluating the relationship between perceived family functioning and BSS. Owing to previous research focused on the discrepancies between patient-family perceptions of family functioning (Keitner et al., 1987), three separate indicators of family functioning were evaluated: adolescent-rated FAD, caregiver-rated FAD, and the mean difference between adolescent- and caregiver-rated FAD (discrepancy score). The association between each family functioning indicator and BSS was evaluated alone (simple linear regression) as well as while controlling for negative affect (multiple linear regression). The third step involved a set of logistic regression analyses using suicide attempt status as the outcome variable. Again adolescent, caregiver, and discrepancy FAD scores were evaluated in relation to attempt status alone (simple logistic regression) and when adding negative affect into the model (multiple logistic regression). All regression analyses included only data from participants with no missing data for suicidal indicators.

\section{Results}

Table 1 summarizes descriptive data for self-report measures used. A paired samples t-test indicated that adolescent FAD scores $(M=2.45, S D=0.62)$ were significantly higher (indicating more perceived dysfunction) than caregiver FAD scores $(M=2.08, S D=0.44$ ), $t(82)=5.30, \mathrm{p}<0.001$.

Results of all regression analyses are presented in Table 2. Adolescent FAD score was significantly associated with BSS. Multiple linear regression analyses revealed that the positive association between adolescent FAD and BSS remained significant when NASSQ was added into the model. Simple logistic regression analyses yielded a significant positive association between adolescent FAD score and presence of a past suicide attempt. This relationship was no longer significant when NASSQ was added as a covariate. 
Primary analyses were conducted with all available data from adolescents and caregivers. ANOVAs were conducted comparing the FAD, BSS and NASSQ scores of adolescents whose caregivers participated and those whose caregivers did not participate and a chisquare test was conducted to detect any differences in suicide attempt status. No significant differences were observed. Additionally, sensitivity analyses using only those adolescents whose caregivers completed the relevant self-report forms were also conducted. With this smaller sample size $(n=88)$, the relationship between adolescent perception of family functioning was still significant both in the simple linear regression $(F(1,72)=5.71, p=$ $0.02)$ and when controlling for the NASSQ $(F(2,71)=13.45, \mathrm{p}<0.01)$. The relationship between adolescent perceived family functioning and suicide attempt status dropped below significance $($ Wald $=1.83, \mathrm{p}=0.18$, Odds Ratio $=1.77)$ using this subset of the data, likely the result of a decrease in power.

\section{Discussion}

This study found that suicidal adolescents perceived their family functioning to be significantly worse than their caregivers. Furthermore, even after controlling for negative affect, adolescent-rated family functioning significantly predicted suicidal ideation, but not attempt status. Caregiver-rated family functioning and the discrepancy between caregiver and adolescent family functioning ratings were not significantly associated with either suicidal outcome variable either before or after controlling for negative affect.

These results build on existing literature in several ways. First, they support the importance of adolescent perception of family functioning as a predictor of suicidal ideation. Consistent with the findings observed in this study, previous research with adolescents suggests that there is an association between suicidal behavior and family dysfunction (e.g., King et al., 1993; Martin et al., 1995; Brinkman-Sull et al., 2000; Bridge et al., 2006). However, existing literature suggests that when a negative affect variable (e.g., depression severity) is included in the predictive model as a covariate, the predictive value of the variable representing family functioning is no longer significant (King, et al., 1993; Brinkman-Sull, et al., 2000). The present findings suggest that family functioning predicts suicidal ideation even after the variance accounted for by negative affect has been removed. One implication of the present study, therefore, is that intervening on adolescent perception of family functioning may reduce ideation. Given the strong connection between suicidal ideation and attempts, such an intervention may have a subsequent effect on suicidal behavior (Kuo et al., 2001).

Second, findings indicate that adolescents perceived significantly more family dysfunction than their caregivers. Previous research with adults has found a significant discrepancy between perceived family functioning of suicidal patients and family members on the general functioning subscale of the FAD (Keitner, et al., 1987; McDermut, et al., 2001), but to the best of our knowledge, this is the first replication of this finding in an adolescent sample. In the FAD development sample, nonclinical participants' mean FAD score was 1.96 and clinical participants mean FAD score was 2.26 (Epstein et al., 1983). Given this normative data, the difference between the average caregiver and adolescent FAD ratings in this study may be interpreted as being both clinically as well as statistically significant. Adolescents in the present study obtained a mean FAD score of 2.45, which is well above the clinical cutoff implied in Epstein et al. (1983). Mean caregiver FAD ratings (2.06) were closer to those observed by Epstein et al. (1983) in a nonclinical sample (1.96) than in the clinical sample (2.26).

Third, results did not support an association between caregiver-adolescent discrepancy and indictors of suicide. Although the discrepancy variable produced a nearly significant 
association with ideation in a simple linear regression, only adolescent ratings remained significantly predictive of ideation when negative affect was included in the model. Previous research with adults has emphasized a relationship between suicidal behavior and the discrepancy between patient and family perceptions of family functioning (Keitner et al., 1987). The current findings suggest that, while family-patient discrepancy in perceptions of family functioning is consistently observed in suicidal inpatient populations, this discrepancy is not predictive of suicidal ideation or attempt status in adolescents.

More complete case self-report data was available for adolescents than for caregivers. Higher self-report completion rates for adolescents compared to caregivers was expected given that recruitment efforts focused on adolescent participation and the majority of the recruited adolescents were able to complete self-report measures while hospitalized. Sensitivity analyses conducted omitting the adolescents without caregiver self-report data indicated that observed relationships held with the exception of the association between adolescent perceptions of family functioning and attempt status. These findings suggest that differences in the predictive relationship between adolescent FAD ratings and suicidal ideation and parent FAD ratings and suicidal ideation are not likely owing to parents' lower response rate.

There are several limitations to this study. First, our data were drawn exclusively from an inpatient sample selected on the basis of severe suicidal ideation or intent. This may limit generalizability to patients with lower levels of suicidality. Second, assessment of perceived family functioning was limited to a brief self-report measure. Third, selection bias may have occurred insofar as participants had to agree to participate in a six-month, follow-up study of suicidal behavior. Also, only those participants who completed baseline self-report data were included in the present study. Fourth, nearly two thirds of the sample was female. This gender distribution is consistent with findings that females demonstrate more suicide attempts and ideation, but fewer completed suicides than males (Lewinsohn et al., 2001). Finally, this discussion takes a suicide risk factor perspective, but cross-sectional associations found do not necessarily imply causality. Future research with a longitudinal design will be needed to make causal inferences with regard to the relationship between perception of family dysfunction and suicidal behavior.

Nevertheless, this study has a number of strengths and will help guide future investigation. Use of a uniformly suicidal population and a continuous measure of suicidality (the BSS) allowed us to draw conclusions about the predictive nature of perceived family functioning among high-risk patients. The inclusion as a covariate of a measure of negative affect that is highly correlated with depressed mood and anxiety (Ronan et al., 1994) also allowed for the evaluation of unique variance in suicidal ideation accounted for by perceived family functioning. Use of this measure as a covariate, therefore, provides more conservative conclusions than are typically found because depressive and anxious negative affectivity are accounted for in the analyses. Findings indicate a need for further investigation of family factors that produce significant family-patient discrepancies, and the temporal evaluation of how perceived family functioning and suicidality vary together. Finally, our results suggest the importance of assessing adolescent perceptions of family functioning and the need for family-based interventions for adolescents at risk for suicide.

\section{Acknowledgments}

This research was supported by National Institute of Mental Health (NIMH) grant MH069904 to Shirley Yen. The views expressed in this articles are those of the authors and do not necessarily reflect the official position of NIMH. We would like to acknowledge the support of our clinical collaborators, Dr. Dawn Picotte, Dr. Joel Solomon, Dr. Sharon Shephard, and Dr. Gerald Tarnoff, and research staff, Heather Pelletier, Sarah Samways, and Caitlin Melvin. 


\section{References}

Beck AT, Kovacs M, Weissman A. Assessment of suicidal ideation: the Scale for Suicidal Ideation. Journal of Consulting and Clinical Psychology. 1979; 47:343-352. [PubMed: 469082]

Bridge JA, Goldstein TR, Brent DA. Adolescent suicide and suicidal behavior. Journal of Child Psychology and Psychiatry. 2006; 47:372-394. [PubMed: 16492264]

Brinkman-Sull DC, Overholster JC, Silverman E. Risk of future suicide attempts in adolescent psychiatric inpatients at 18-month follow-up. Suicide and Life-Threatening Behavior. 2000; 30:327-340. [PubMed: 11210058]

Byles J, Byrne C, Boyle M, Offord D. Ontario Child Health Study--relability and validity of the general functioning subscale of the McMaster Family Assessment Device. Family Process. 1988; 27:97-104. [PubMed: 3360100]

Centers for Disease Control and Prevention. Unpublished mortality data from the National Center for Health Statistics (NNHS) Mortality Data Tapes. Centers for Disease Control; 2002.

Centers for Disease Control and Prevention. Youth risk behavior surveillance--United States, 2009. Surveillance Summaries, June 4; MMWR. 2010. p. 59Retreived from http://www.cdc.gov/mmwr/ preview/mmwrhtml/ss5905a1.htm

Epstein NB, Baldwin LM, Bishop DS. The McMaster Family Assessment Device. Journal of Marital and Family Therapy. 1983; 9:171-180.

Goldston DB, Daniel SS, Erkanli A, Reboussin BA, Mayfield A, Frazier PH, Treadway SL. Psychiatric diagnoses as contemporaneous risk factors for suicide attempts among adolescents and young adults: developmental changes. Journal of Consulting and Clinical Psychology. 2009; 77:281-290. [PubMed: 19309187]

Holi MM, Pelkonen M, Karlsson L, Kiviruusu O, Ruuttu T, Heila H, Tuisku V, Marttunen M. Psychometric properties and clinical utility of the Scale for Suicidal Ideation (SSI) in adolescents. BMC Psychiatry. 2005; 5:8. [PubMed: 15691388]

Kabacoff R, Miller I, Bishop D, Epstein N, Keitner G. A psychometric study of the McMaster Family Assessment Device in psychiatric medical and nonclinical samples. Journal of Family Psychology. 1990; 3:431-439.

Kaufman J, Bormaher B, Brent D, Rau U, Flynn C, Moreci P, Williamson D, Ryan N. Schedule for affective disorders and schizophrenia for school-aged children-present and lifetime version (KSADS-PL): initial reliability and validity data. Journal of the American Academy of Child and Adolescent Psychiatry. 1997; 36:980-988. [PubMed: 9204677]

Keitner GI, Miller IW, Fruzzetti AE, Epstein NB, Bishop DS, Norman WH. Family functioning and suicidal behavior in psychiatric inpatients with major depression. Psychiatry. 1987; 50:242-255. [PubMed: 3659212]

King CA, Segal HG, Naylor M, Evans T. Family functioning and suicidal behavior in adolescent inpatients with mood disorders. Journal of the American Academy of Child and Adolescent Psychiatry. 1993; 32:1198-1206. [PubMed: 8282665]

Kuo W, Gallo JJ, Tien AY. Incidence of suicide ideation and attempts in adults: the 13-year follow-up of a community sample in Baltimore, Maryland. Psychological Medicine. 2001; 31:1181-1191. [PubMed: 11681544]

Lewinsohn PM, Rhode P, Seeley JR, Baldwin CL. Gender differences in suicide attempts from adolescence to young adulthood. Journal of the American Academy of Child \& Adolescent Psychiatry. 2001; 40:427-434. [PubMed: 11314568]

Liu X, Gentzler AL, Tepper P, Kiss E, Kothencne VO, Tamas Z, Vetro A, Kovacs M. Clinical features of depressed children and adolescents with various forms of suicidality. Journal of Clinical Psychiatry. 2006; 67:1442-1450. [PubMed: 17017833]

Martin G, Rotaries P, Pearce C, Allison S. Adolescent suicide, depression and faily dysfunction. Acta Psychiatrica Scandinavica. 1995; 92:336-344. [PubMed: 8619337]

McDermut W, Miller IW, Solomon D, Ryan CE, Keitner GI. Family Functioning and Suicidality in Depressed Adults. Comprehensive Psychiatry. 2001; 42:96-104. [PubMed: 11244144]

Miller IW, Epstein NB, Bishop DS, Keitner GI. The McMaster Family Assessment Device: reliability and validity. Journal of Marital and Family Therapy. 1985; 11:345-356. 
Miranda R, Scott M, Hicks R, Wilcox HC, Munfakh JLH, Shaffer D. Suicide attempt characteristics, diagnoses, and future attempts: comparing multiple attempters to single attempters and ideators. Journal of the American Academy of Child and Adolescent Psychiatry. 2008; 47:32-40. [PubMed: 18174823]

Muehlenkamp JJ, Gutierrex PM. Risk for suicide attempts among adolescents who engage in nonsuicidal self-injury. Archives of Suicide Research. 2007; 11:69-82. [PubMed: 17178643]

Nrugham L, Larsson B, Sund AM. Predictors of Suicidal acts across aolescence: Influences of familial, peer and individual factors. Journal of Affective Disorders. 2008; 109:35-45. [PubMed: 18096243]

Ronan KR, Kendall PC, Rowe M. Negative Affectivity in Children: Development and Validation of a Self-Statement Questionaire. Cognitive Therapy and Research. 1994; 18:509-528.

Shaffer D, Gould MS, Fisher P, Trautman P, Moreau D, Kleinman M, Flory M. Psychiatric diagnosis in child and adolescent suicide. Archives of General Psychiatry. 1996; 53:339-348. [PubMed: 8634012]

Uebelacker LA, Beevers CG, Battle CL, Strong D, Keitner GI, Ryan CE, Solomon DA, Miller IW. Family functioning in bipolar I disorder. Journal of Family Psychology. 2006; 20:701-704. [PubMed: 17176206]

Weinstock LM, Miller IW. Psychosocial predictors of mood symptoms 1 year after acute phase treatment of bipolar I disorder. Comprehensive Psychiatry. 2010; 51:497-503. [PubMed: 20728007]

Yen, S.; Weinstock, L.; Andover, M.; Sheets, E.; Selby, EA.; Spirito, A. Prospective predictors of adolescent suicidality: Six month post hospitalization follow-up. under review 


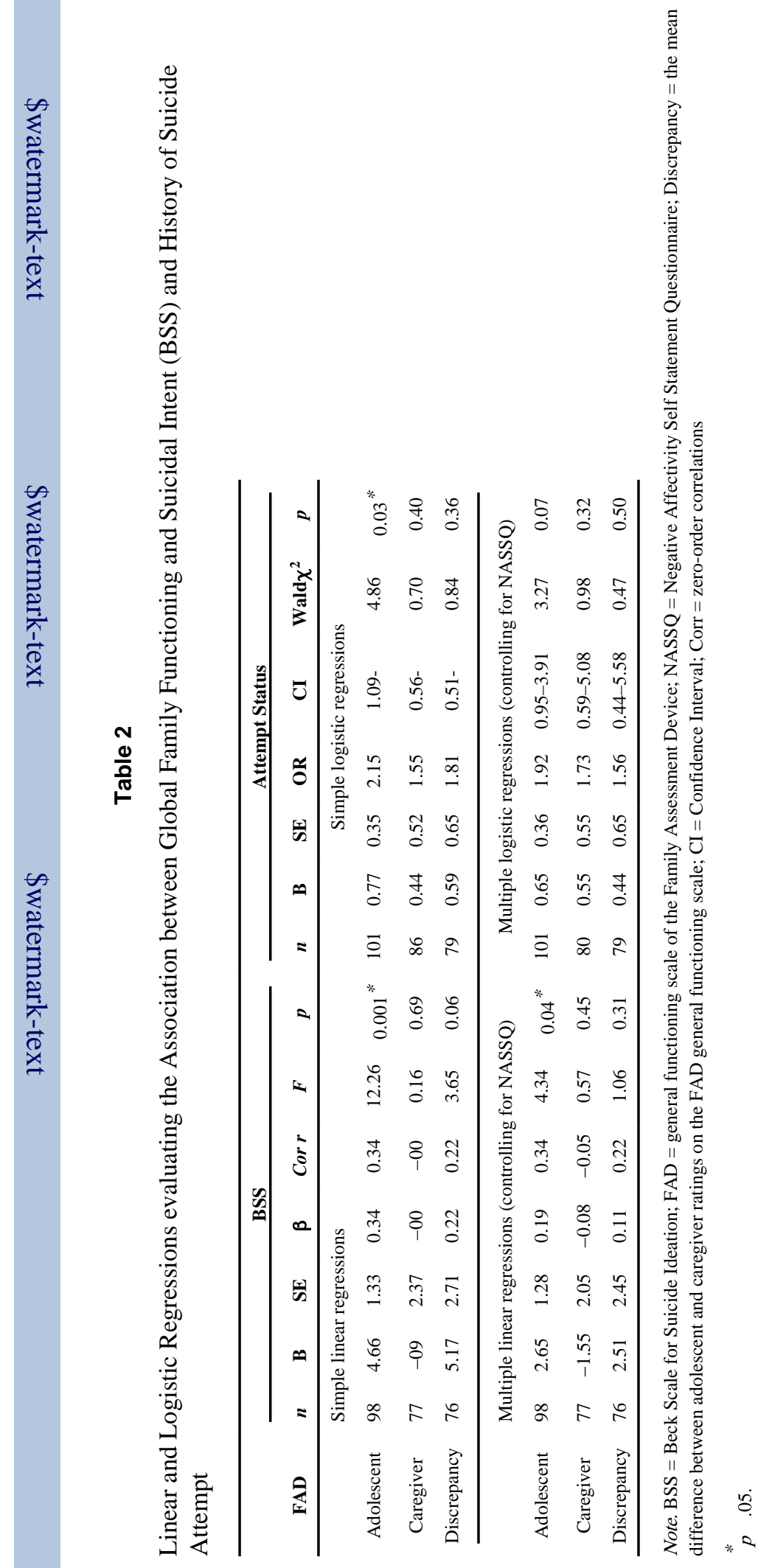

